\title{
Function of inducible nitric oxide synthase in the regulation of cervical cancer cell proliferation and the expression of vascular endothelial growth factor
}

\author{
JING DONG, MINGJUN CHENG and HONG SUN \\ Obstetrics and Gynecology Hospital, Shanghai Medical College, Fudan University, Shanghai 200011, P.R. China
}

Received August 9, 2012; Accepted November 1, 2013

DOI: $10.3892 / \mathrm{mmr} .2013 .1838$

\begin{abstract}
Inducible nitric oxide synthase (iNOS) is the key enzyme in NO synthesis and exhibits a high expression in numerous types of malignant tumors. Previous studies have demonstrated that iNOS promotes the production of tumor blood vessels by catalyzing the synthesis of additional NO. Certain experimental evidence has suggested that iNOS may regulate the levels of vascular endothelial growth factor (VEGF), as the level of VEGF may be induced by iNOS during endothelial cell migration and angiogenesis. In this study, it was observed that $\mathrm{SiHa}$ and HeLa cells exhibit depressed proliferation levels following the knockdown of iNOS with a lentivirus. In addition, NO levels demonstrated a correlation with VEGF levels in the cell culture supernatant.
\end{abstract}

\section{Introduction}

Cervical cancer is one of the most common malignant cancers in gynecology (1-3). Recently, the incidence rate of the disease has increased and has demonstrated an increasing trend in younger females. However, the developmental process of the tumor is complex and involves numerous factors at various stages $(4,5)$. Previous studies have demonstrated that angiogenesis was the foundation for tumor growth $(6,7)$ and that NO is one of the most secondary messengers and is capable of promoting the process (1).

Vascular endothelial growth factor (VEGF) is considered to be one of the most significant of the 30 cytokines whose function it is to promote vascular production (8). VEGF increases cancer cell growth by promoting the cleavage and proliferation of tumor cells by binding to the tyrosinase receptor (9-11).

Correspondence to: Professor Hong Sun, Obstetrics and Gynecology Hospital, Shanghai Medical College, Fudan University, 419 Fangxie Road, Shanghai 200011, P.R. China

E-mail: sun321hong@163.com

Key words: inducible nitric oxide synthase, cervical cancer cell, proliferation, vascular endothelial growth factor
Inducible nitric oxide synthase (iNOS) is a key enzyme in NO synthesis and has a high expression in numerous types of malignant tumors. Previous studies have demonstrated that iNOS promotes the production of tumor blood vessels by catalyzing the synthesis of additional NO $(9,12)$. Experimental evidence has suggested that iNOS may emerge as a VEGF enhancer, due to the fact that the VEGF level is capable of being induced by iNOS in endothelial cell migration and angiogenesis (13). However, the correlation between iNOS and VEGF in cervical cancer remains unclear $(11,14)$.

\section{Materials and methods}

Cell culture. Two human cervical carcinoma cell lines, Hela and SiHa, which exhibit relatively low metastatic capability, were obtained from the China Center for Type Culture Collection (Wuhan, China). Cells were all cultured in Dulbecco's modified Eagle's medium supplemented with $10 \%$ fetal bovine serum (FBS; Gibco, Grand Island, NY, USA) in an atmosphere of $5 \% \mathrm{CO}_{2}$ and $95 \%$ air. SiHa and HeLa cells were plated in 6-well plates.

Quantitative polymerase chain reaction ( $q P C R)$. Total RNA was extracted from $1 \times 10^{6}$ human $\mathrm{SiHa}$ and $\mathrm{HeLa}$ cells using TRIzol (Invitrogen Life Technologies, Carlsbad, CA, USA). Aliquots ( $1 \mathrm{~g}$ ) of RNA were reverse transcribed to cDNA and aliquots (4 $\mu \mathrm{l}$ ) of cDNA were used as a template for qPCR using an ABI7900HT PCR system (Applied Biosystems, Carlsbad, CA, USA) according to the manufacturer's instructions. The primers were as follows: Forward: 5'-TGGAGAGAAACTGAAGAAATCG-3' and reverse 5'-GTACCTGAATTTGTTGTTGAGC-3' for iNOS; forward: 5'-GCTGTGAAGCCAGACAGC-3' and reverse: 5'-GGCAAGTCACCCTGCTGA-3' for VEGF; and forward: 5'-CTCCATCCTGGCCTCGCTGT-3' and reverse: 5'-GCTGTCACCTTCACCGTTCC-3' for $\beta$-actin (ACTB). All experiments were performed in triplicate, with each reading taken three times. Average and standard deviations were subsequently calculated.

Western blotting. A nuclear extraction kit (Chemicon International, Temecula, CA, USA) was used to isolate cytoplasmic proteins. Proteins, $50 \mu \mathrm{g}$, from various groups were boiled for $5 \mathrm{~min}$ in sample buffer and were subsequently 
separated in sodium dodecyl sulphate-polyacrylamide gel electrophoresis (SDS-PAGE) (10-15\%) and transferred onto a polyvinylidene fluoride (PVDF) membrane (Bio-Rad Laboratories, Hercules, CA, USA). Nonspecific reactivity was blocked using $5 \%$ bovine serum albumin in a Tris-buffered saline (TBS) with Tween-20 buffer (100 ml 10\% TBS, $10 \mathrm{ml}$ $10 \%$ Tween-20 final $0.1 \% \mathrm{v} / \mathrm{v}$, dissolved with $890 \mathrm{ml}$ double distilled- $\mathrm{H}_{2} \mathrm{O}$ ) and subsequently incubated with primary antibody mouse anti-VEGF (sc-1836, 1:200), rabbit anti-iNOS (sc-650, 1:200) or rabbit anti-actin (sc-130656, 1:500) antibodies (all from Santa Cruz Biotechnology, Santa Cruz, CA, USA) overnight at $4^{\circ} \mathrm{C}$ and incubated with horseradish peroxidase (HRP)-conjugated secondary antibodies (Santa Cruz Biotechnology). Bands were detected using the Supersignal West Dura Extended Duration Substrate (Pierce, Rockford, IL, USA), and quantified using the Chemi-doc gel quantification system (Bio-Rad). All data were normalized to $\beta$-actin. Western blotting experiments were repeated at least twice in order to confirm the results.

Enzyme-linked immunosorbent assay (ELISA) and NO detect assay (Griess method). The quantity of VEGF released was measured by a sandwich ELISA. ELISA plates (Cat. No: 655001; Greiner, Bio-One, Frickenhausen, Germany) were coated with $100 \mu \mathrm{l}$ of $2 \mu \mathrm{g} / \mathrm{ml}$ anti-VEGF165 (R\&D Systems, Minneapolis, MN, USA) antibody in phosphate-buffered saline (PBS) for $12 \mathrm{~h}$ at $4^{\circ} \mathrm{C}$. The plates were washed with PBS containing $0.1 \%$ Tween 20 (TPBS) and incubated for $1 \mathrm{~h}$ at $25^{\circ} \mathrm{C}$ with $200 \mu \mathrm{l} /$ well of $1 \%$ bovine serum albumin (SigmaAldrich, St. Louis, MO, USA) in PBS. The conditioned medium, or various concentrations of recombinant human VEGF, were incubated for $2 \mathrm{~h}$ at $25^{\circ} \mathrm{C}$ and washed four times with TPBS. Following incubation for $2 \mathrm{~h}$ at $25^{\circ} \mathrm{C}$ with $100 \mu \mathrm{l}$ of $0.2 \mu \mathrm{g} / \mathrm{ml}$ biotinylated anti-VEGF antibody, the plates were washed and incubated for a further $45 \mathrm{~min}$ with $100 \mu \mathrm{l} \mathrm{HRP-conjugated}$ streptavidin (Vector Laboratories, Burlingame, CA, USA). After washing, the plates were developed by adding $50 \mu \mathrm{l}$ tetramethylbenzidine (Sigma-Aldrich) and the reaction was stopped by adding $50 \mu \mathrm{l} 2 \mathrm{NH}_{2} \mathrm{SO}_{4}$. The absorbance at $450 \mathrm{~nm}$ was measured with a Tecan 96-well plate reader (Tecan, Untersbergstrasse, Austria). The NO level was detected by supernatant absorbance at $540 \mathrm{~nm}$, according to the Griess method (11).

Cell proliferation and cell apoptosis assays. Cell proliferation assays were performed with cell counting kit-8 (CCK-8; Dojindo, Kumamoto, Japan). SiHa and HeLa cells treated with nonsense sequence (SCR) or lentiviruses expressing iNOS-targeted shRNA (iNOS-SH) were seeded into 96-well plates at $5 \times 10^{3}$ per well in a $100 \mu \mathrm{l}$ cell culture medium and maintained at $37^{\circ} \mathrm{C}$ in a humidified incubator containing $5 \% \mathrm{CO}_{2}$ for $24 \mathrm{~h}$. One hundred microlitres of $2 \% \mathrm{FBS}$ medium was used as a control. After 96 cultures, $10 \mu \mathrm{l}$ CCK- 8 solution was added to the triplicate wells and incubated for $3 \mathrm{~h}$. Subsequently, the absorbance at $450 \mathrm{~nm}$ was measured in order to calculate the number of vital cells in each well. Cell proliferation is represented as the mean percentage \pm standard deviation of absorbance. Cell apoptosis assays were performed by flow cytometry with propidium iodide (PI) and Annexin V-fluorescein isothiocyanate (FITC) once the cells had been digested with trypsin, washed and harvested.
Short hairpin RNA (shRNA) interference, lentivirus production and infection. iNOS-SH shRNA was generated from two different annealed oligonucleotides (target 1: 5'-CCGGCCAGAAGCAGAATGTGACATCTC GAGTGGTCACATTCTGCTTCTGGTTTTTG-3' and 5'-AATTCAAAAACCAGAAGCAGAATGTGACATCTCGA GATGGTCACATTCTGCTTCTGG-3'; target 2: 5'-CCGGGAAGCGGTAACAAAGGAGATACTCGAGTATC T C C T T T G T T A C C G C T T C T T T T T G - $3{ }^{\prime}$ a n d 5'-AATTCAAAAAGAAGCGGTAACAAAGGAGATACTCGAG TATCTCCTTTGTTACCGCTTC-3'; and target 3: 5'-CCGGCGAGGACTATTTCTTTCAGCTCTCGAGAGCT GAAAGA AATAGTCCTCGTTTTTG-3' and 5'-AA TTCAAAAACGAGGACTATTTCTTTCAGCTCTCGAGAGCT GAAAGAAATAGTCCTCG-3') that were inserted into the AgeI and EcoRI sites of SCR vector purchased from Addgene; the human iNOS target sequence is underlined. The control shRNA was purchased from Addgene. All target sequences were designed and verified as specific for iNOS by Blast searching against the human genome. The resulting plasmids, iNOS-SH1, iNOS-SH2 and iNOS-SH3, were sequenceverified.

To obtain a recombinant lentivirus, HEK-293T cells were cotransfected with the package, envelope and iNOS-SH constructs. The virus-containing supernatant was harvested following $60 \mathrm{~h}$ cotransfection and concentrated by ultracentrifugation at 100,000 x g for $2 \mathrm{~h}$ with an Avanti J-30I (Beckman Coulter, Miami, FL, USA). For infection, the viral stock was supplemented with $8 \mu \mathrm{g} / \mathrm{ml}$ polybrene.

Tumorigenicity assays in nude mice. All studies involving animals were approved by the Ethics Committee of Obstetrics and Gynecology Hospital of Fudan University (Shanghai, China). Five- to six-week-old female athymic BALB/c nude mice were purchased from Sino-British Sippr/BK Lab Animal Ltd, Co. (Shanghai, China). iNOS-SH and SCR-infected HeLa cells $\left(1 \times 10^{6}\right)$ were suspended in $100 \mu$ l PBS and were subcutaneously injected into the posterior flank of 4-5 week-old female nude mice. Tumor size measurement was performed every three days over the course of 4 weeks and the tumor volume was estimated using the formula: Volume $=$ length $\mathrm{x}$ width ${ }^{2} \mathrm{x} 0.5$. The comparison of tumor volumes between the iNOS-SH and vehicle control groups was performed using paired t-tests.

Immunohistochemisty (IHC). Tissues were fixed in $10 \%$ buffered formalin fixative at room temperature overnight. Samples were subsequently deparaffinized in xylene, rehydrated through graded ethanol, quenched for endogenous peroxidase activity in $0.3 \%$ hydrogen peroxide and processed for antigen retrieval by microwave heating in $10 \mathrm{mM}$ citrate buffer ( $\mathrm{pH}$ 6.0) using antibodies against iNOS, VEGF, and myeloperoxidase (MPO) (Dako, Carpinteria, CA, USA). Immunostaining was performed according to the manufacturer's instructions using the ChemMate Dako EnVision Detection kit, Peroxidase/DAB, Rabbit/Mouse (DakoCytomation, Glostrup, Denmark), which resulted in a brown precipitate at the antigen site.

Statistical analysis. All results are expressed as the mean \pm standard error of the mean. Differences were analyzed 
A

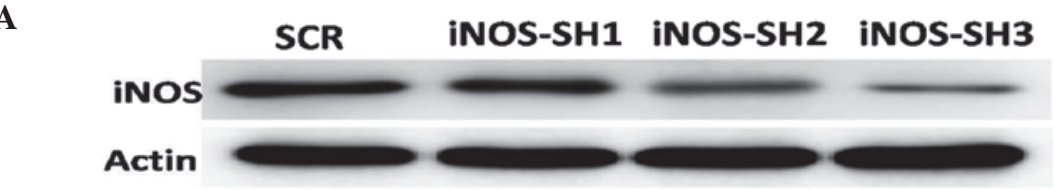

B

D

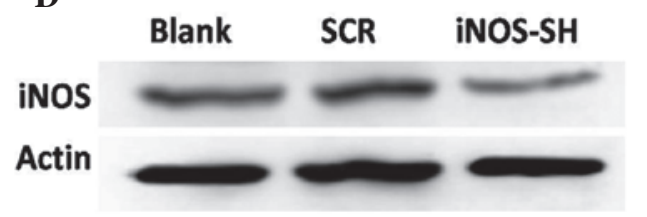

C

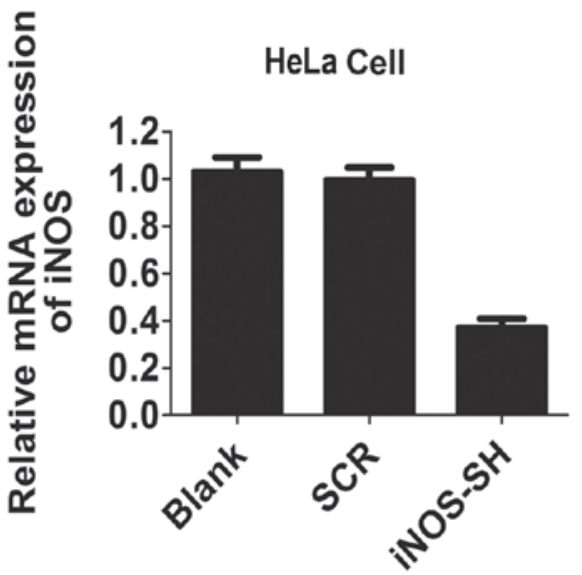

E

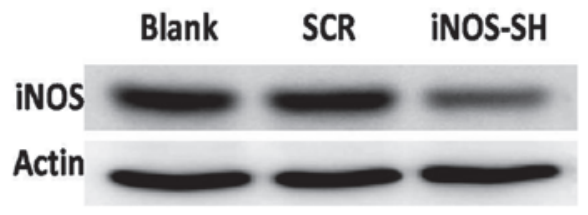

Figure 1. Characterisation of antisense iNOS cell lines in vitro. (A) HeLa cells that were stably transfected with control or iNOS short hairpin RNA and were analyzed by western blotting with antibodies against iNOS and actin. (B) iNOS expression in SiHa cell lines treated with blank, scramble (SCR) and iNOS-SH, determined by quantitative polymerase chain reaction. Results are provided as the mean \pm standard error of the mean (SEM) of triplicates of three separate experiments, ${ }^{*} \mathrm{P}<0.05$, Student's t-test. (C) iNOS expression in HeLa cell lines treated with blank, SCR and iNOS-SH, determined by qPCR. Results are provided as the means \pm SEM of triplicate of three separate experiments, ${ }^{*} \mathrm{P}<0.05$, Student's t-test. (D) iNOS expression in SiHa cell lines treated with blank, SCR and iNOS-SH, determined by western blotting. (E) iNOS expression in HeLa cell lines treated with blank, SCR and iNOS-SH, determined by western blotting. Decreased iNOS expression occurred in the iNOS-SH-SiHa and iNOS-SH-HeLa cell lines. * $\mathrm{P}<0.05$, compared with SCR. iNOS, inducible nitric oxide synthase; SCR, scramble; iNOS-SH, iNOS-targeted shRNA; SEM, standard error of the mean.

by Student's t-test with SPSS 15.0 software, (SPSS, Chicago, IL, USA). $\mathrm{P}<0.05$ was considered to indicate a statistically significant difference.

\section{Results}

Inhibition of iNOS expression in SiHa and HeLa cells. In order to determine the function of iNOS in the development of cervical cancer, a number of SiHa and HeLa cells were not infected or infected with lentiviruses expressing a 20 base fragment of human shRNA and control scramble, respectively. The efficacy of this fragment in inhibiting the expression of iNOS in these cell lines was determined by western blotting, and $\mathrm{SH} 3$ was selected as the iNOS shRNA of the three shRNAs for its high knockdown efficiency (Fig. 1A). According to the results of western blotting and qPCR, the iNOS protein product from the SiHa and HeLa cells was significantly different between the experimental and control groups following iNOS stable knockdown $(\mathrm{P}<0.05)$. In cells expressing iNOS-shRNA, there was significant inhibition of iNOS expression (60\% mRNA knockdown in SiHa cells, and 75\% mRNA knockdown in HeLa cells; $>70 \%$ protein knockdown efficacy in the two cell lines) (Fig. 1)
Effect of inhibiting iNOS expression on tumor growth in vitro and in vivo. To test the function of iNOS in cervical cancer growth, cell proliferation was investigated following iNOS knockdown. As expected, the number of SiHa and HeLa cells decreased markedly under iNOS-SH downregulation, compared with the blank and scramble groups (Fig. 2A and B). However, data from flow cytometry revealed a statistically significant increase in the levels of SiHa and HeLa apoptosis (Fig. 2C and D). The experimental evidence reveals the critical role of iNOS in SiHa and HeLa growth in vitro. In contrast to their growth in vitro, when $\mathrm{SiHa}$ and HeLa cells were inoculated into the flanks of nude mice, the iNOS-SH HeLa cells growth rate in vivo was significantly slower compared with that of the control cells (Fig. 3A and B). Tumors derived from iNOS-SH SiHa cells exhibited a growth rate similar to that of iNOS-SH HeLa tumors. HeLa tumors became palpable and measurable 10 days after cell inoculation compared with $\mathrm{SiHa}$ tumors, which could be measured 13 days following inoculation. Following 20 days of growth, the mean tumor size of HeLa tumors was half that of the control tumors. Inhibition of iNOS expression in HeLa tumors was confirmed by IHC analysis iNOS (Fig. 3C and D) and VEGF (Fig. 3E and F) of tumors with or without iNOS knockdown. 
A

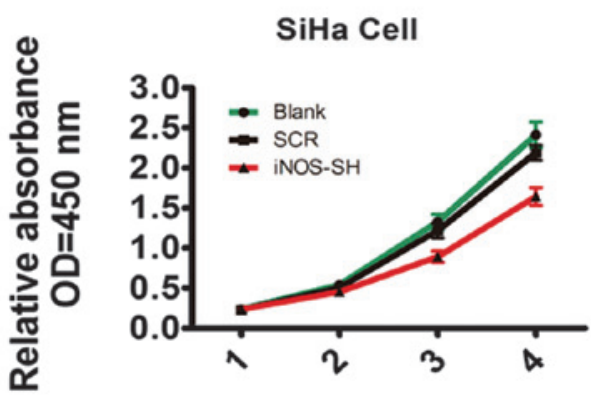

B

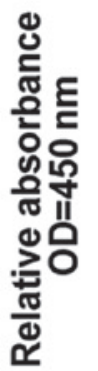

HeLa Cell

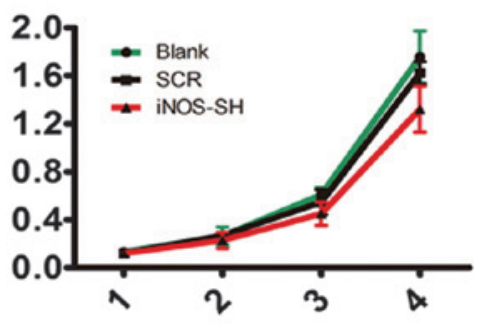

Time (Day)

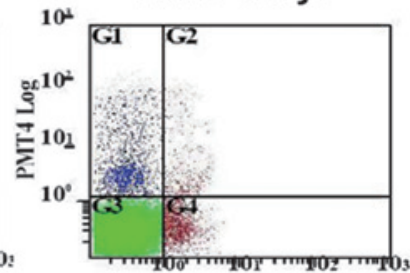

iNOS-SH

D
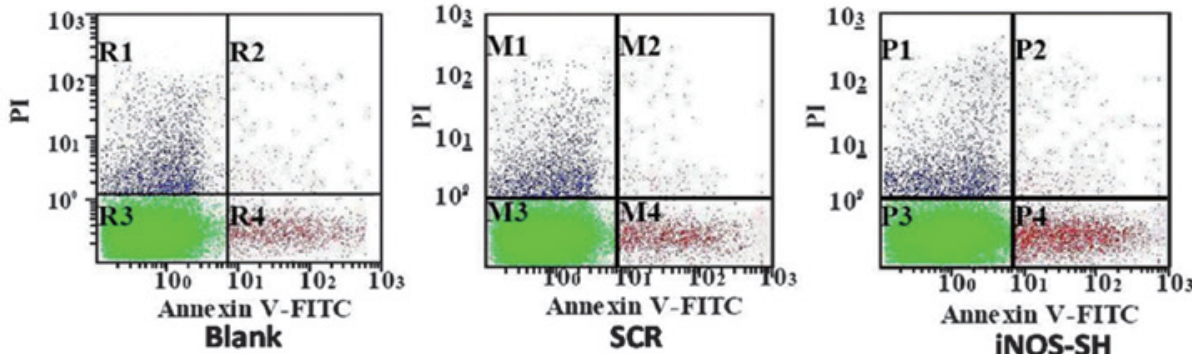

$\mathbf{E}$

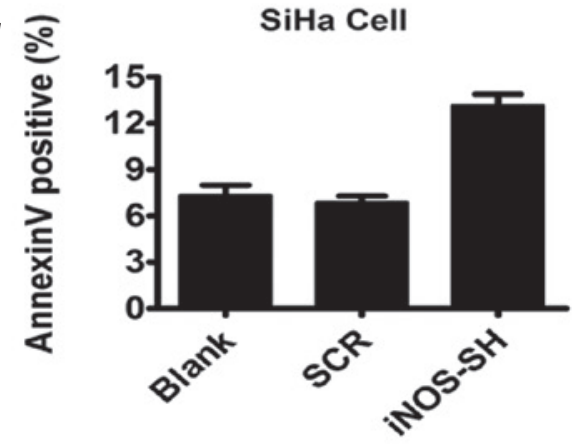

$\mathbf{F}$

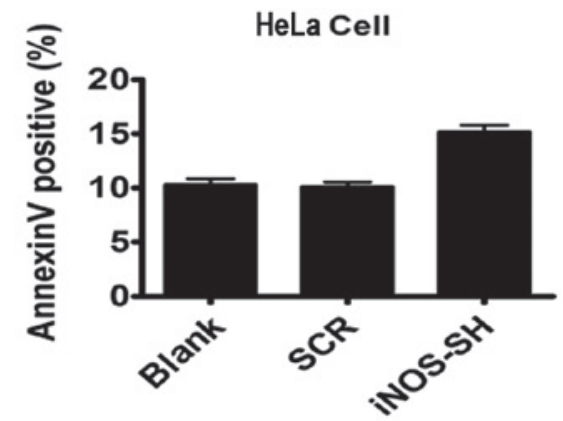

Figure 2. Effect of iNOS RNAi on tumor growth in vitro and in vivo. (A and B) Normalized growth rates of SiHa or HeLa cell lines with or without iNOS knockdown, data points are presented as the mean \pm SEM. The growth rate of iNOS-SH treated cell lines are significantly slower. (C and D) Normalized apoptosis rates of cells derived from $\mathrm{SiHa}$ or HeLa cell lines. Data points are the mean $\pm \mathrm{SEM}$. The apoptosis rate of iNOS-SH treated cell lines was considered to be significant. (E and F) Statistics of SiHa and HeLa cell apoptosis, showing significantly increased level of Annxin V-positive cells in iNOS-SH cells. Data points are the mean \pm SEM. "P<0.05, compared with SCR. iNOS, inducible nitrix oxide synthase; SEM, standard error of the mean; SCR, scramble; iNOS-SH, iNOS-targeted shRNA.

Decreased NO and VEGF level in supernatant and cells in iNOS-knockdown SiHa and HeLa culture. NO has been demonstrated to stimulate and inhibit VEGF expression in a cell-dependent manner and is a potent modulator of vascular development and tumor progression. In in vitro studies, the iNOS-shRNA group demonstrated that the two cell lines produced lower levels of VEGF compared with the control group. The inhibition of iNOS with lentivirus-based-RNAi for $24 \mathrm{~h}$ resulted in a significant 1.5 -fold downregulation of $\mathrm{NO}$ in the supernatant of SiHa and HeLa cell lines (Fig. 4A and B). Furthermore, the concentration of VEGF in the same supernatant was markedly reduced in the iNOS-shRNA group, compared with the control group, as determined by western blotting (Fig. 4C and D).

iNOS increases VEGF levels in SiHa and HeLa cell cultures. To assess whether NO is a mediator for the reduction of VEGF in the supernatant and iNOS-SH treated cells, sodium nitroprusside (SN), an exogenous NO donor, was applied to stimulate cells. The mRNA level of iNOS and VEGF in cells incubated in media with or without SN (DMSO, 0.25, 0.5 and $1 \mathrm{mM}$ ) were assessed (Fig. 5A and B). In all groups, the VEGF mRNA level (Fig. 5B) demonstrated a significant increase with treatment with a high concentration of $\mathrm{SN}(0.5 \mathrm{mM})$, 

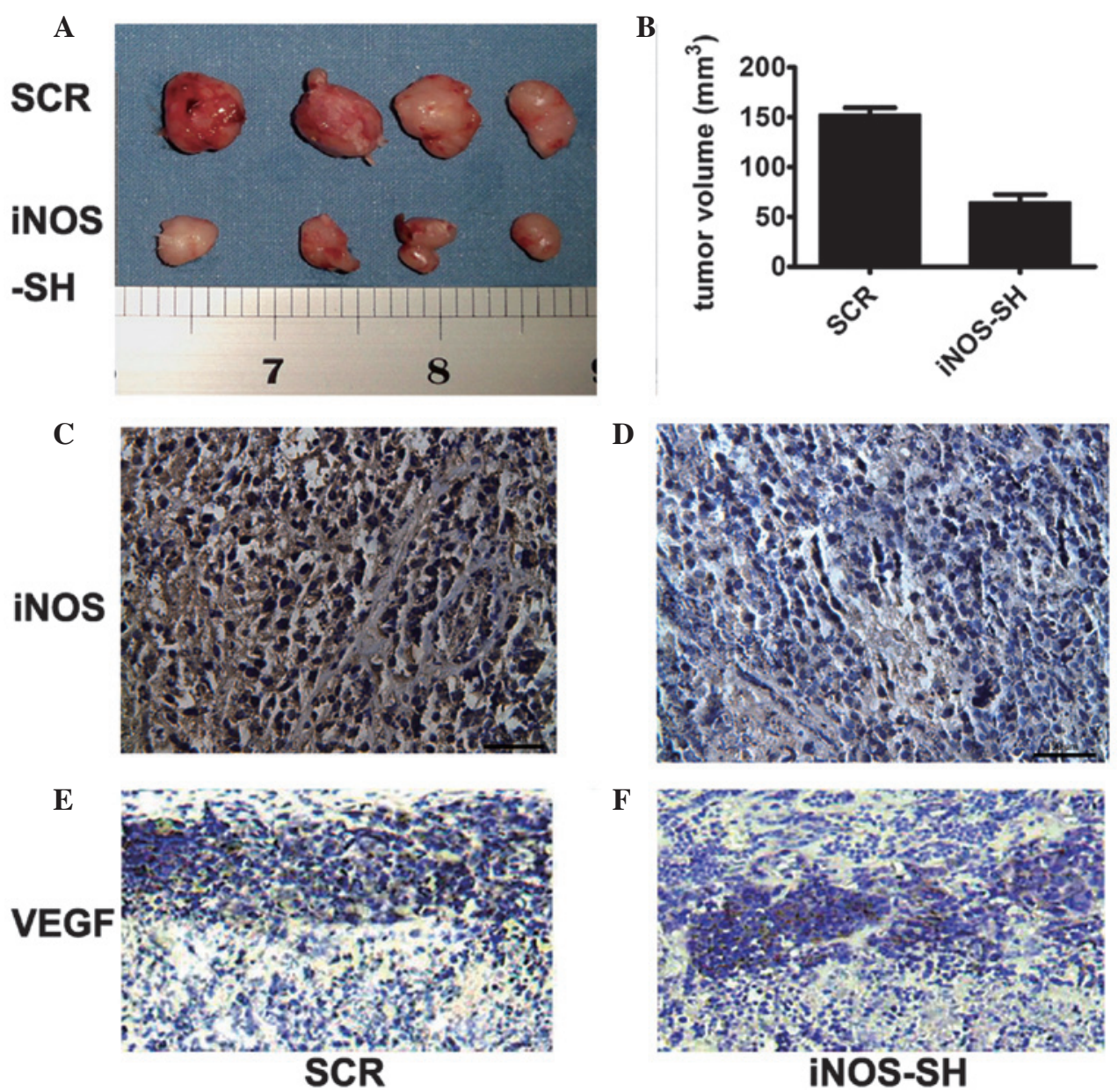

$\mathbf{F}$

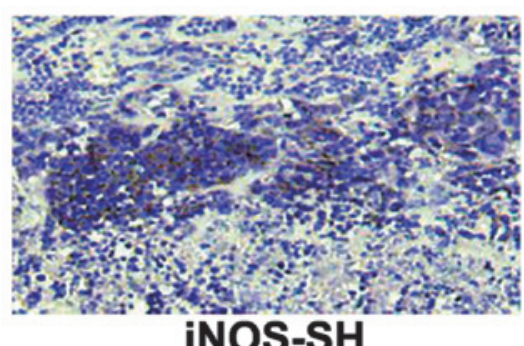

Figure 3. Effect of iNOS RNAi on HeLa and SiHa growth assessed by immunohistochemistry. (A) Representative image of iNOS effect in HeLa tumor growth (B) Tumor weight statistics of (A). (C and D) Representative image of iNOS expression in HeLa tumor growth. (E and F) show represented image of VEGF expression in HeLa tumor growth. DAB was stained as a control. "P<0.05, compared with SCR. Original magnification, x200. iNOS, inducible nitric oxide synthase; VEGF, vascular endothelial growth factor; SCR, scramble; iNOS-SH, iNOS-targeted shRNA.
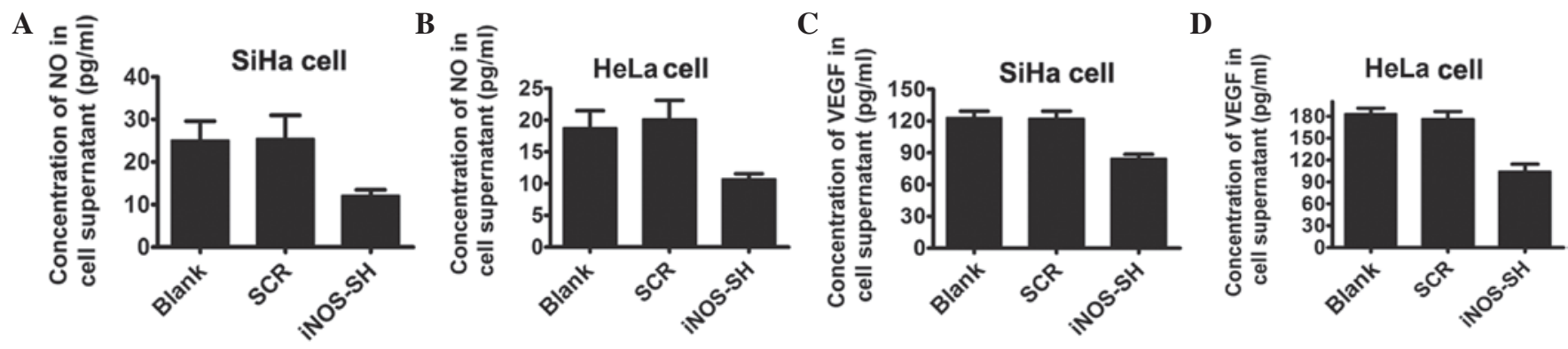

Figure 4. The effect of iNOS on NO production and the secretion of VEGF. (A and B) Differences in supernatant NO levels among the experimental groups. $\mathrm{NO}$ analysis was performed on supernatants from the cell cultures collected 2 days after their infection with iNOS-SH. Control cells were transfected with or without scramble shRNA. Data are presented as the mean \pm standard error of the mean ${ }^{*} \mathrm{P}<0.05$. (C and D) Cell supernatant was harvested and used for VEGF concentration quantification with the human VEGF enzyme-linked immunosorbent assay kit. "P $<0.05$, compared with SCR. iNOS, inducible nitric oxide synthase; VEGF, vascular endothelial growth factor; SCR, scramble, iNOS-SH, iNOS-targeted shRNA.

without changing the iNOS level, which may mimic the effect of iNOS. However, in SiHa and HeLa cells, SN increased the supernatant concentration of $\mathrm{NO}$ in a dose-dependent manner. The fact that $\mathrm{SN}$ increased the supernatant concentration of NO in iNOS-SH SiHa and HeLa cells reveals that SN induced NO production in an iNOS-independent way (Fig. 5C and D). Furthermore, in SiHa and HeLa cells the stable knockdown of iNOS-SH, the protein levels of VEGF and iNOS peaked at
$0.5 \mathrm{mM}$ SN treatment, but exhibited a smaller increase with $1 \mathrm{mM} \mathrm{SN}$ (Fig. 5E and F).

In conclusion, the data indicated that the knockdown of iNOS based on depressed lentivirus proliferation of SiHa and HeLa, may be mimicked by applying an extrogenous NO donor. Furthermore, iNOS is capable of regulating the proliferation of cervical cancer cells and the expression of VEGF by regulating the concentration of NO. 
A

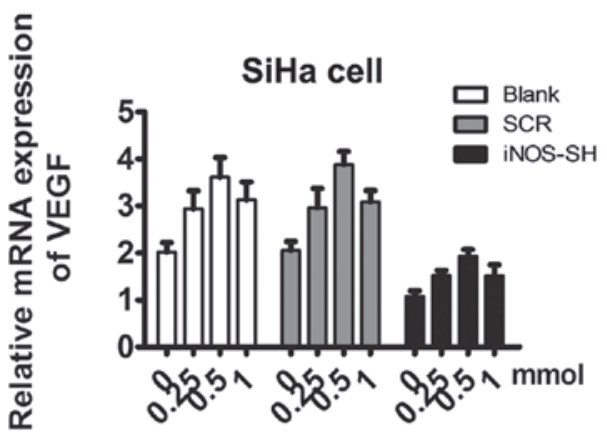

C

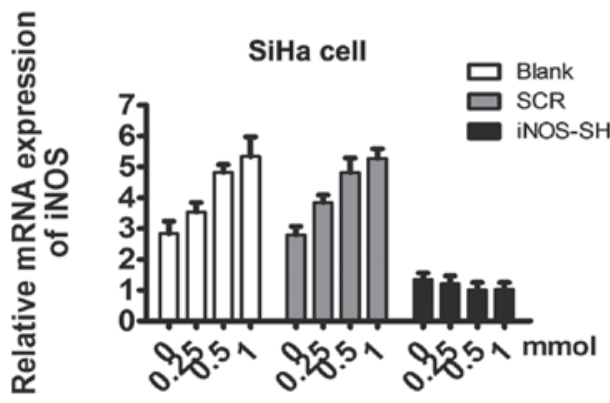

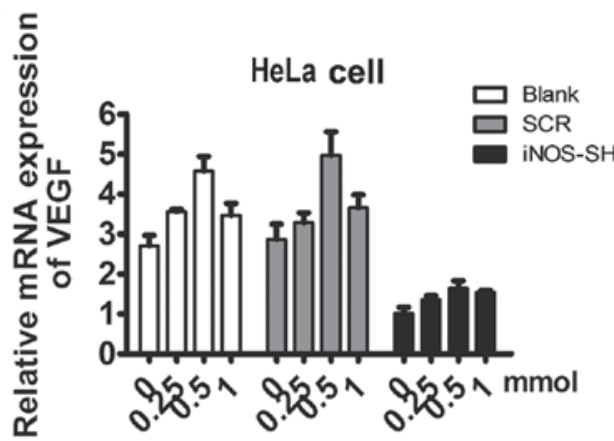

D

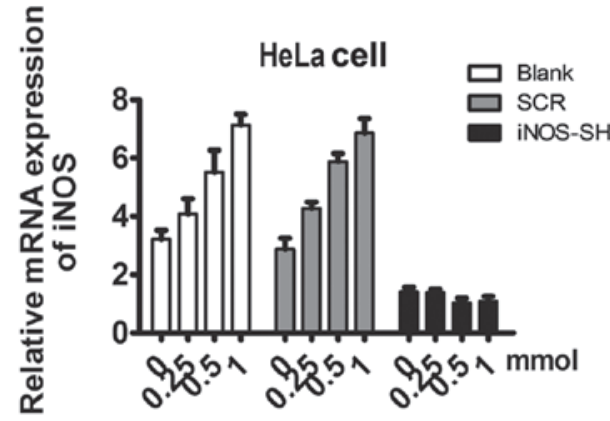

E

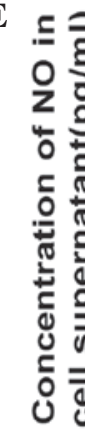

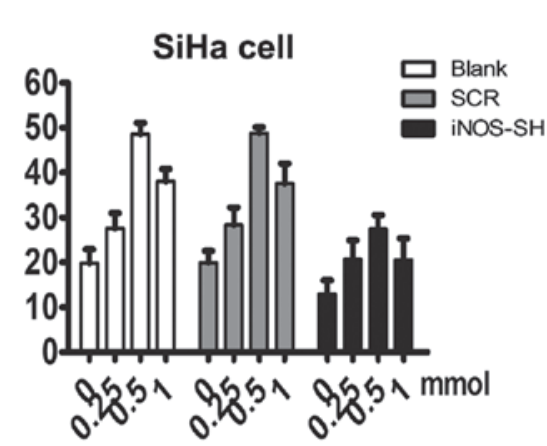

F

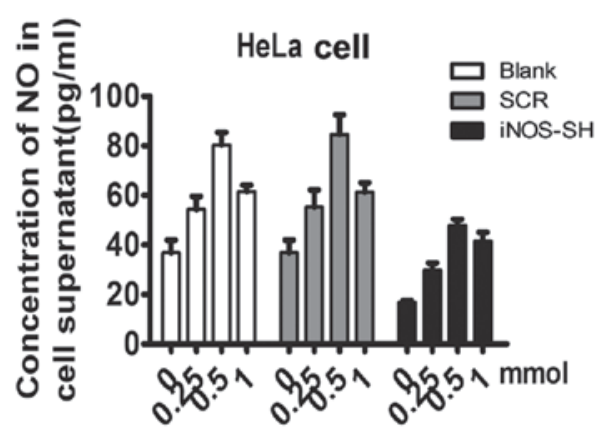

6

VEGF

iNOS

Actin

SN (mmol)

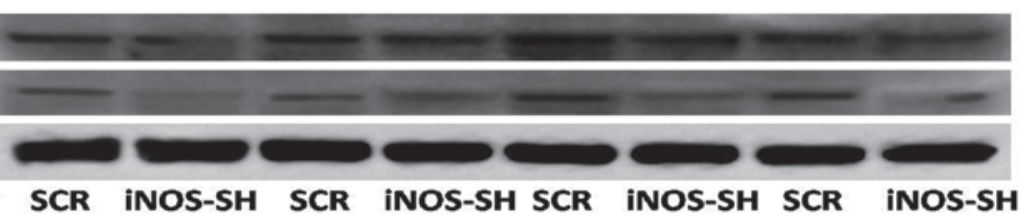

0.25

0.25

0.5

0.5

1.0

1.0

H

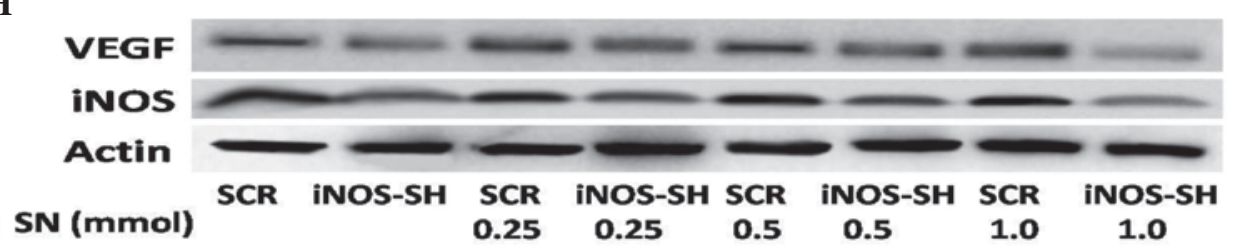

Figure 5. Effect of extrogenous NO on VEGF in SiHa and HeLa cells. (A and B) Quantitation of iNOS mRNA level obtained from SiHa and HeLa treated with SN among each experiment group. Cells harvested following stimulation for $24 \mathrm{~h}$ with $0.25,0.5$ or $1 \mathrm{mM}$ SN, DMSO was applied as the control. (C and D) Quantitation of VEGF mRNA level obtained from SiHa and HeLa treated with SN among each experiment group. Cells harvested after stimulation for $24 \mathrm{~h}$ with $0.25,0.5$ or $1 \mathrm{mM} \mathrm{SN}$, DMSO was applied as the control. (E and F) The supernatant concentration of NO treated with or without SN (DMSO, $0.25,0.5$ and $1 \mathrm{mM})(\mathrm{G}$ and $\mathrm{H})$ Protein levels of VEGF and iNOS in cells treated with SN. Results are provided as the means \pm SEM. "P $<0.05$, Student's t-test. NO, nitric oxide; VEGF, vascular endothelial growth factor; iNOS, inducible nitric oxide synthase; SN, sodium nitroprusside; DMSO, dimethylsulfoxide : SCR, scramble; iNOS-SH, iNOS-targeted shRNA.

\section{Discussion}

In recent years, the incidence rate of cervical cancer has increased markedly, with an increasing trend in young females. However, the mechanisms underlying the disease remain unknown.
Several factors have been implicated in tumor development. One of the most prominent cytokines is VEGF, which induces sprouting angiogenesis, increases blood vessel permeability and maintains tumor vessel integrity (15). VEGF expression has been shown to be a survival factor for blood vessels in SiHa (14). 
iNOS and VEGF are important in tumor growth. The level of iNOS is correlated with that of VEGF. One of the most important mechanisms is that angiogenesis is induced by iNOS, which is capable of synthesizing more NO, regulating VEGF levels in tumors. NO is capable of inducing VEGF expression and mediating the angiogenic effects of VEGF (16). Therefore it was hypothesized that the decline in growth and vessel perfusion of $\mathrm{SiHa}$ and HeLa tumor cells may correlate with decreased VEGF expression. Notably, analyses of VEGF expression and production in tumor cells and explants following RNAi of iNOS revealed a correlation between VEGF and NO levels. This, coupled with previously published data, suggests that NO produced from iNOS activity is important for the maintenance of tumor blood vessel tone and function and further highlights the different roles of NOS isotypes and their tissue/cell type of origin in vivo (17).

NO production is induced by numerous angiogenic stimuli and is an important extracellular and intracellular signaling molecule synthesized from arginine by NOS. Of the three NOS isoforms, including iNOS, endothelial (eNOS) and neuronal NOS, iNOS specifically produces the greatest quantities of NO and is associated with a number of pathologies (18). Although their precise roles remain unclear, the activites of iNOS and eNOS have been implicated in tumor progression and angiogenesis, and their effectiveness appears to be dependent on their activity and distribution, the concentration and duration of exposure to NO and the intrinsic sensitivity of cells to NO (19). The effect of altered NO levels on tumor growth and angiogenesis has also been exploited through pharmacological manipulation with either NO donors or NOS inhibitors.

In the present study, it was observed that iNOS knockdown results in the decreased proliferation of SiHa and HeLa cells and the concentration of VEGF in the supernatant, which means that iNOS regulates the growth of cervical cancer cells in a VEGF-dependent process. Further studies are required in order to confirm that $\mathrm{SN}$ is capable of rescuing the iNOS-induced decrease.

In conclusion, it was demonstrated i) Using a lentivirus-delivered RNAi approach that iNOS is a key modulator of tumor growth and angiogenesis; ii) that the level of VEGF correlates with the expression of iNOS in SiHa and HeLa cells, which may be mediated by the level of NO; and iii) that the level of NO generated in the tumor is lower than the concentration at which apoptosis occurs, which promotes growth and angiogenesis.

\section{Acknowledgements}

This work was supported by The National Natural Science Foundation of China (81272877) and Cancer Foundation of China.

\section{References}

1. Walboomers JM, Jacobs MV, Manos MM, et al: Human papillomavirus is a necessary cause of invasive cervical cancer worldwide. J Pathol 189: 12-19, 1999.
2. Bosch FX, Manos MM, Munoz N, et al: Prevalence of human papillomavirus in cervical cancer: a worldwide perspective. International biological study on cervical cancer (IBSCC) Study Group. J Natl Cancer Inst 87: 796-802, 1995.

3. Dürst M, Gissmann L, Ikenberg H and zur Hausen H: A papillomavirus DNA from a cervical carcinoma and its prevalence in cancer biopsy samples from different geographic regions. Proc Natl Acad Sci USA 80: 3812-3815, 1983.

4. Bosch FX, Lorincz A, Muñoz N, Meijer CJ and Shah KV: The causal relation between human papillomavirus and cervical cancer. J Clin Pathol 55: 244-265, 2002.

5. Morris M, Eifel PJ, Lu J, et al: Pelvic radiation with concurrent chemotherapy compared with pelvic and para-aortic radiation for high-risk cervical cancer. N Engl J Med 340: 1137-1143, 1999.

6. Isacson C, Kessis TD, Hedrick L and Cho KR: Both cell proliferation and apoptosis increase with lesion grade in cervical neoplasia but do not correlate with human papillomavirus type. Cancer Res 56: 669-674, 1996.

7. Tsang RW, Fyles AW, Li Y, et al: Tumor proliferation and apoptosis in human uterine cervix carcinoma I: correlations between variables. Radiother Oncol 50: 85-92, 1999.

8. Lo HW, Hsu SC, Ali-Seyed M, et al: Nuclear interaction of EGFR and STAT3 in the activation of the iNOS/NO pathway. Cancer Cell 7: 575-589, 2005.

9. Kroll $\mathrm{J}$ and Waltenberger J: VEGF-A induces expression of eNOS and iNOS in endothelial cells via VEGF receptor-2 (KDR). Biochem Biophys Res Commun 252: 743-746, 1998.

10. Ichinoe M, Mikami T, Shiraishi H and Okayasu I: High microvascular density is correlated with high VEGF, iNOS and COX-2 expression in penetrating growth-type early gastric carcinomas. Histopathology 45: 612-618, 2004.

11. Zhang J and Peng B: NF-kappaB promotes iNOS and VEGF expression in salivary gland adenoid cystic carcinoma cells and enhances endothelial cell motility in vitro. Cell Prolif 42: 150-161, 2009.

12. Saura M,Zaragoza C, McMillan A, et al: An antiviral mechanism of nitric oxide: inhibition of a viral protease. Immunity 10: 21-28, 1999.

13. Kostourou V, Cartwright JE, Johnstone AP, et al: The role of tumour-derived iNOS in tumour progression and angiogenesis. Br J Cancer 104: 83-90, 2011.

14. Benjamin LE, Hemo I and Keshet E: A plasticity window for blood vessel remodelling is defined by pericyte coverage of the preformed endothelial network and is regulated by PDGF-B and VEGF. Development 125: 1591-1598, 1998.

15. Holash J, Davis S, Papadopoulos N, et al: VEGF-Trap: a VEGF blocker with potent antitumor effects. Proc Natl Acad Sci USA 99: 11393-11398, 2002.

16. Feng CW, Wang LD, Jiao LH, Liu B, Zheng S and Xie XJ: Expression of p53, inducible nitric oxide synthase and vascular endothelial growth factor in gastric precancerous and cancerous lesions: correlation with clinical features. BMC Cancer 2: 8 , 2002.

17. Chiarugi V, Magnelli L and Gallo O: Cox-2, iNOS and p53 as play-makers of tumor angiogenesis (review). Int J Mol Med 2: 715-724, 1998.

18. Martínez-Estrada OM, Rodríguez-Millán E, González-De Vicente E, Reina M, Vilaró S and Fabre M: Erythropoietin protects the in vitro blood-brain barrier against VEGF-induced permeability. Eur J Neurosci 18: 2538-2544, 2003.

19. Fukumura D and Jain RK: Tumor microvasculature and microenvironment: targets for anti-angiogenesis and normalization. Microvasc Res 74: 72-84, 2007. 